\title{
The Rand and block distances of pairs of set partitions
}

\author{
Frank Ruskey $^{1 \star}$ and Jennifer Woodcock ${ }^{1}$ \\ Dept. of Computer Science, University of Victoria, CANADA
}

\begin{abstract}
The Rand distances of two set partitions is the number of pairs $\{x, y\}$ such that there is a block in one partition containing both $x$ and $y$, but $x$ and $y$ are in different blocks in the other partition. Let $R(n, k)$ denote the number of distinct (unordered) pairs of partitions of $n$ that have Rand distance $k$. For fixed $k$ we prove that $R(n, k)$ can be expressed as $\sum_{j} c_{k, j}\left(\begin{array}{c}n \\ j\end{array}\right) B_{n-j}$ where $c_{k, j}$ is a non-negative integer and $B_{n}$ is a Bell number. For fixed $k$ we prove that there is a constant $K_{n}$ such that $R\left(n,\left(\begin{array}{l}n \\ 2\end{array}\right)-k\right)$ can be expressed as a polynomial of degree $2 k$ in $n$ for all $n \geq K_{n}$. This polynomial is explicitly determined for $0 \leq k \leq 11$. The block distance of two set partitions is the number of elements that are not in common blocks. We give formulae and asymptotics based on $N(n)$, the number of pairs of partitions with no blocks in common. We develop an $O(n)$ algorithm for computing the block distance.
\end{abstract}

\section{Introduction and Motivation}

In statistics, particularly as it is applied to cluster analysis, it is sometimes useful to have a measure of the difference between two set partitions [4]. The Rand distance is one such measure, and was introduced in Rand [8]. In this paper we will initiate a combinatorial study of the properties of the Rand distance, taken over all unordered pairs of partitions of an $n$-set. We will also introduce another measure, which we call the block distance, and determine some of its properties. For example, we will determine an exact expression for the number of pairs of partitions that have no blocks in common. Furthermore, we will show how to compute the block distance efficiently.

The Rand distance of two set partitions is the number of unordered pairs $\{x, y\}$ such that there is a block in one partition containing both $x$ and $y$, but $x$ and $y$ are in different blocks in the other partition. We use $\mathcal{R}(P, Q)$ to denote the Rand distance between two set partitions $P$ and $Q$. For example, $\mathcal{R}(\{\{1,2\},\{3\}\},\{\{1\},\{2,3\}\})=2$ (the pairs are $\{1,2\}$ and $\{2,3\})$ and $\mathcal{R}(\{\{1,2,3\}\},\{\{1\},\{2\},\{3\}\})=3$ (the pairs are $\{1,2\},\{1,3\}$, and $\{2,3\})$. In general, if $P$ and $Q$ are partitions of an $n$-set, then $0 \leq \mathcal{R}(P, Q) \leq\left(\begin{array}{c}n \\ 2\end{array}\right)$.

Let $R(n, k)$ be the number of distinct (unordered) pairs of partitions of an $n$-set that have Rand distance $k$. See Table 2 in Section 3.1. This table was computed from exhaustive computer listings of all partitions of $\{1,2, \ldots, n\}$ up

\footnotetext{
* Research supported in part by NSERC
} 
to $n=11$. The column sums are $\left(\begin{array}{c}B_{n} \\ 2\end{array}\right)$. Note that the numbers for fixed $n$ are not unimodal in general.

We define the block distance $\mathcal{B}(P, Q)$ between two partitions of $n$ as the number of elements in the blocks that are not common to both $P$ and $Q$. For example,

$$
\mathcal{B}(\{\{1,2\},\{3\}\},\{\{1\},\{2\},\{3\}\})=2
$$

since the only block that is common to both partitions is $\{3\}$ and there are 2 elements in the remaining blocks. By $B(n, k)$ we denote the number of pairs of partitions of $n$ that have block distance $k$. See Table 1 in Section 2. The Rand distance can be cleverly computed using a linear number of arithmetic operations; see Filkov and Skiena [2] and we will show that the block distance is also efficiently computable.

Organizationally, we will finish this section by giving some background on set partitions. In the succeeding two sections, we discuss first the block distance and then the Rand distance. The focus is mainly on the elucidation of some enumerative results along with a clever $O(n)$ algorithm for computing the block distance.

\subsection{Background on set partitions}

A partition of a set $S$ is collection of disjoint subsets of $S$, say $\left\{S_{1}, S_{2}, \cdots, S_{k}\right\}$ whose union is $S$. Each $S_{i}$ is referred to as a block. The number of partitions of an $n$-set into $k$ blocks is the Stirling number (of the second kind), which is denoted as $\left\{\begin{array}{l}n \\ k\end{array}\right\}$. We use $[n]$ to denote $\{1,2, \ldots, n\}$.

In the computer, partitions are usually represented by restricted growth strings. We assume that the blocks of a partition $X$ are numbered $S_{1}, S_{2}, \ldots, S_{k}$ according to the size of the smallest element in each block. That is, $S_{1}$ contains $1, S_{2}$ contains the smallest element not in $S_{1}$, and so on. Then the restricted growth string $r[1 . . n]$ of $X$ is defined by taking $r[i]$ to be the distance of the block containing $i$. The Gray code algorithms for generating restricted growth strings developed in [9] and discussed in [5] were used to generate the numbers in Tables 1 and 2; as each string was generated the $O(n)$ algorithms for computing the Rand distance and the block distance were applied.

The $n$-th Bell number, $B_{n}$, is the total number of partitions of an $n$-set, irrespective of block size. Thus $B_{n}=\sum_{k}\left\{\begin{array}{l}n \\ k\end{array}\right\}$. The exponential generating function (egf) of the Bell numbers is well-known (e.g., Stanley [10], pg. 34)) to be

$$
B(z)=\sum_{n \geq 1} B_{n} \frac{z^{n}}{n !}=e^{e^{z}-1} .
$$

The number of pairs of partitions is $\left(\begin{array}{c}B_{n} \\ 2\end{array}\right)$. For $n=1,2,3, \ldots, 10$ these numbers are

$0,1,10,105,1326,20503,384126,8567730,223587231,6725042325$.

They give the row sums in Tables 1 and 2 . 
We use several times a generalization of the fact that if $f(z)=\sum_{n>0} f_{n} z^{n} / n$ ! is the egf of a sequence $f_{n}$, then $z f(z)$ is the egf of the sequence $n f_{n-1}$. See Knuth, Graham, Patashnik [3], page 350. Furthermore, for $k \geq 0$,

$$
\begin{aligned}
z^{k} f(z) & =\sum_{n \geq k} n(n-1) \cdots(n-k+1) f_{n-k} \frac{z^{n}}{n !} \\
& =k ! \sum_{n \geq 0}\left(\begin{array}{l}
n \\
k
\end{array}\right) f_{n-k} \frac{z^{n}}{n !} .
\end{aligned}
$$

Thus $k !\left(\begin{array}{l}n \\ k\end{array}\right) f_{n-k}$ is the $n$-th coefficient of $z^{k} f(z)$.

\section{The Block Distance}

Recall that the block distance $\mathcal{B}(P, Q)$ of two partitions of $n$ is the number of elements in the blocks that are not common to both $P$ and $Q$, and that $B(n, k)$

\begin{tabular}{|c|c|c|c|c|c|c|c|c|}
\hline$n \backslash k$ & 2 & 3 & 4 & 5 & 6 & 7 & 8 & 9 \\
\hline 2 & 1 & & & & & & & \\
\hline 3 & 3 & 7 & & & & & & \\
\hline 4 & 12 & 28 & 65 & & & & & \\
\hline 5 & 50 & 140 & 325 & 811 & & & & \\
\hline 6 & 225 & 700 & 1950 & 4866 & 12762 & & & \\
\hline 7 & 1092 & 3675 & 11375 & 34062 & 89334 & 244588 & & \\
\hline 8 & 5684 & 20384 & 68250 & 227080 & 714672 & 1956704 & 5574956 & \\
\hline
\end{tabular}
is the number of pairs of partitions of $n$ that have block distance $k$. See Table 1 .

Table 1. The values of $B(n, k)$ for $1 \leq k \leq n \leq 9$.

Let $N(n)=B(n, n)$; this is the number of unordered pairs of partitions that have no blocks in common. The numerical values of $N(n)$, for $0 \leq n \leq 10$, are

$$
0,0,1,7,65,811,12762,244588,5574956,148332645,4538695461 \text {. }
$$

Determining $N(n)$ for $i=1, \ldots, n$ is sufficient to determine $B(n, k)$ since, by direct combinatorial considerations,

$$
B(n, k)=N(k)\left(\begin{array}{l}
n \\
k
\end{array}\right) B_{n-k} .
$$

We also note that

$$
\left(\begin{array}{c}
B_{n} \\
2
\end{array}\right)=\sum_{k=0}^{n} B(n, k)=\sum_{k=0}^{n} N(k)\left(\begin{array}{l}
n \\
k
\end{array}\right) B_{n-k} .
$$


Letting $N(z)$ be the egf of the $N(n)$ numbers, from (4) we obtain the equation

$$
P(z):=\sum_{n \geq 0}\left(\begin{array}{c}
B_{n} \\
2
\end{array}\right) \frac{z^{n}}{n !}=N(z) e^{e^{z}-1} .
$$

And thus

$$
N(z)=P(z) e^{1-e^{z}} .
$$

The egf $e^{1-e^{z}}$ is known; it is the egf of the "complementary Bell numbers" (OEIS A000587). The complementary Bell numbers, $C_{n}$, for $n=0,1,2, \ldots, 14$ are

$$
1,-1,0,1,1,-2,-9,-9,50,267,413,-2180,-17731,-50533,110176 .
$$

It is known that

$$
C_{n}=\sum_{k=0}^{n}(-1)^{k}\left\{\begin{array}{l}
n \\
k
\end{array}\right\} .
$$

Thus, from (5) we get a "closed-form" formula for $N(n)$, namely

$$
N(n)=\sum_{j=0}^{n}\left(\begin{array}{l}
n \\
j
\end{array}\right) C_{j}\left(\begin{array}{c}
B_{n-j} \\
2
\end{array}\right)=\sum_{j=0}^{n}\left(\begin{array}{l}
n \\
j
\end{array}\right)\left(\begin{array}{c}
B_{n-j} \\
2
\end{array}\right) \sum_{k=0}^{j}(-1)^{k}\left\{\begin{array}{l}
j \\
k
\end{array}\right\} .
$$

\subsection{Linear Time Algorithm to Compute the Block Distance}

In this subsection we present a linear time algorithm to compute the block distance of two partitions.

Closely related to the restricted growth string, we define the block string, $b[1 . . n]$, of $P$ as follows: $b[i]$ is the smallest element in the block containing $i$. Every block string has the characterizing property that $b[1]=1$, and for $i>1$,

$$
b[i] \in\{i, b[1], b[2], \ldots, b[i-1]\} .
$$

It is relatively simple to convert a restricted growth string into the corresponding block string in $O(n)$ time.

The following code takes as input a restricted growth function $r[1 . . n]$ and returns the corresponding block string $b[1 . . n]$. It uses a temporary array $m[1 . . n]$ that maintains the invariant $b[i]=m[r[i]]$.

$$
\begin{aligned}
& \text { for } i \in\{1,2, \ldots, n\} \text { do } m[i]:=0 ; \\
& \text { for } i:=1,2, \ldots, n \text { do } \\
& \quad \text { if } m[r[i]]=0 \text { then } m[r[i]]:=i \text {; } \\
& \quad b[i]:=m[r[i]] ;
\end{aligned}
$$

Before describing the algorithm for computing the block distance, we encourage the reader to consider the following small example. Suppose

$$
P=\{1\}\{2\}\{3,4\}\{5,7\}\{6\}, \quad Q=\{1,2\}\{3,4,6\}\{5,7\} .
$$


Then the restricted growth strings for $P$ and $Q$ are

$$
r P=1,2,3,3,4,5,4, \quad r Q=1,1,2,2,3,2,3
$$

and the block strings are

$$
p=1,2,3,3,5,6,5, \quad q=1,1,3,3,5,3,5 .
$$

Comparing the elements, we find that the blocks labelled 1,2,3, and 6 are not common to $P$ and $Q$ and that the block labelled 5 is common to $P$ and $Q$. Since there are 5 elements in blocks $1,2,3$, and 6 , the block distance of $P$ and $Q$ is 5 .

The algorithm maintains a boolean array $C[1 . . n]$ with the property that, upon termination, $C[i]$ is true if $i$ is in a block common to $P$ and $Q$, and is false otherwise. The block distance is thus equal to the number of entries in this array that are false.

The algorithm makes two passes over $p$, one pass over $q$, and one pass over $C$. Consider $p[i]$ and $q[i]$; there are three mutually exclusive cases: (a) $p[i] \neq q[i]$ and $i$ is not in a common block, (b) $p[i]=q[i]$ and $i$ is in a common block, and (c) $p[i]=q[i]$ and $i$ is not in a common block. (Because we are using the block string and not the restricted growth string, it is not possible that $p[i] \neq q[i]$ and $i$ is in a common block.) In the first pass we test only for case (a). In the second pass we (indirectly) distinguish cases (b) and (c).

The key observation is this: If $i$ is not in a common block and $p[i]=q[i]$, then there is some value $j \neq i$ such that $j$ is in the same block as $i$ in $P$ but is in a different block than $i$ in $Q$, or vice-versa. In other words, $p[i]=p[j] \neq q[j]$ or $p[j] \neq q[j]=q[i]$. Thus, in the first pass $C[p[j]]$ and $C[q[j]]$ were set to false.

So on the second pass, we test whether $C[p[i]]$ is false to determine whether $i$ is in a common block or not. On the final pass, we find the block distance by counting the number of false values in $C$. Below is the code in detail.

$$
\begin{aligned}
& \text { for } i \in\{1,2, \ldots, n\} \text { do } C[i]:=\text { true } \\
& \text { for } i:=1,2, \ldots, n \text { do } \\
& \quad \text { if } p[i] \neq q[i] \text { then } C[p[i]]:=C[q[i]]:=\text { false; } \\
& \text { for } i:=1,2, \ldots, n \text { do } \\
& \quad \text { if } \neg C[p[i]] \text { then } C[i]:=\text { false; } \\
& c:=0 ; \\
& \text { for } i \in\{1,2, \ldots, n\} \text { do } \\
& \quad \text { if } \neg C[i] \text { then } c:=c+1 ; \\
& \text { return }(c) ;
\end{aligned}
$$

\section{Results on the Rand distance}

Now recall that $\mathcal{R}(P, Q)$ is the number of unordered pairs $\{x, y\}$ such that there is a block in one partition containing both $x$ and $y$, but $x$ and $y$ are in different blocks in the other partition, and that $R(n, k)$ is the number of distinct (unordered) pairs of partitions of an $n$-set that have Rand distance $k$. See Table 2. Let $R(n)$ be the sum of the Rand distance over all unordered pairs of partitions. 


\begin{tabular}{|c|c|c|c|c|c|c|c|c|}
\hline$k \backslash n \mid$ & 34 & 5 & 6 & 7 & 8 & 9 & 10 & 11 \\
\hline 1 & 312 & 50 & 225 & 1092 & 5684 & 31572 & 186300 & 1163085 \\
\hline 2 & 6301 & 150 & 780 & 4200 & 23772 & 141624 & 887220 & 5835060 \\
\hline 3 & 1322 & 2801 & 1720 & 10885 & 69272 & 452508 & 3060360 & 21482340 \\
\hline 4 & 243 & $300 \vdots$ & 3360 & 25200 & 183960 & 1341648 & 9883440 & 74471760 \\
\hline 5 & & 2403 & 3426 & 42672 & 391356 & 3266172 & 26969040 & 222185304 \\
\hline 6 & & 2204 & 4100 & 56889 & 696178 & 7234374 & 67288830 & 612903720 \\
\hline 7 & & 602 & 2400 & 60165 & 941088 & 12259368 & 141778440 & 1469224350 \\
\hline 8 & & 152 & 2700 & 57750 & 1182888 & 18992502 & 256463820 & 3164268690 \\
\hline 9 & & 101 & 1075 & 46585 & 1150520 & 23324140 & 399874640 & 5762811670 \\
\hline 10 & & 1 & 471 & 31374 & 1165416 & 28129626 & 547907454 & 9538994388 \\
\hline 11 & & & 150 & 24528 & 815640 & 26605908 & 670419540 & 13513772745 \\
\hline 12 & & & 35 & 14140 & 780570 & 26190612 & 742419510 & 18112131840 \\
\hline 13 & & & 45 & 4725 & 413840 & 21568932 & 744780330 & 20675910420 \\
\hline 14 & & & 15 & 1890 & 369180 & 17119818 & 701747010 & 23653643310 \\
\hline 15 & & & 1 & 1302 & 178080 & 13040280 & 607809750 & 22677991578 \\
\hline 16 & & & & 252 & 115780 & 8948079 & 520591950 & 22923998460 \\
\hline 17 & & & & 210 & 43512 & 6244308 & 377521875 & 19287053775 \\
\hline 18 & & & & 140 & 20734 & 3679032 & 312082260 & 17554312490 \\
\hline 19 & & & & 105 & 6860 & 2431044 & 198307620 & 13495597225 \\
\hline 20 & & & & 21 & 7098 & 1250109 & 158606532 & 11143736604 \\
\hline 21 & & & & 1 & 3508 & 640908 & 87210930 & 8029798920 \\
\hline 22 & & & & & 574 & 315828 & 63688410 & 6035010960 \\
\hline 23 & & & & & 840 & 197568 & 33243120 & 4254456690 \\
\hline 24 & & & & & 665 & 57288 & 25703205 & 2872892550 \\
\hline 25 & & & & & 476 & 46116 & 11343906 & 1924619235 \\
\hline 26 & & & & & 210 & 30366 & 6764940 & 1215058680 \\
\hline 27 & & & & & 28 & 25732 & 3272500 & 789847190 \\
\hline 28 & & & & & 1 & 7695 & 2003805 & 453548480 \\
\hline 29 & & & & & & 4104 & 1532340 & 306871290 \\
\hline 30 & & & & & & 2226 & 757080 & 177358500 \\
\hline 31 & & & & & & 3780 & 211410 & 112440900 \\
\hline 32 & & & & & & 2205 & 212625 & 53211510 \\
\hline 33 & & & & & & 1344 & 198345 & 35497935 \\
\hline 34 & & & & & & 378 & 138600 & 16793040 \\
\hline 35 & & & & & & 36 & 82512 & 13781493 \\
\hline 36 & & & & & & 1 & 21080 & 10664335 \\
\hline 37 & & & & & & & 16200 & 6744100 \\
\hline 38 & & & & & & & 15750 & 2483415 \\
\hline 39 & & & & & & & 14910 & 1445565 \\
\hline 40 & & & & & & & 13545 & 802164 \\
\hline 41 & & & & & & & 7245 & 1320165 \\
\hline 42 & & & & & & & 3270 & 860640 \\
\hline 43 & & & & & & & 630 & 580965 \\
\hline 44 & & & & & & & 45 & 215325 \\
\hline 45 & & & & & & & 1 & 104313 \\
\hline 46 & & & & & & & & 62205 \\
\hline 47 & & & & & & & & 103950 \\
\hline 48 & & & & & & & & 70455 \\
\hline 49 & & & & & & & & 74250 \\
\hline 50 & & & & & & & & 45045 \\
\hline 51 & & & & & & & & 21945 \\
\hline 52 & & & & & & & & 7095 \\
\hline 53 & & & & & & & & 990 \\
\hline 54 & & & & & & & & 55 \\
\hline 55 & & & & & & & & 1 \\
\hline
\end{tabular}

Table 2. The values of $R(n, k)$ for $2 \leq n \leq 11$ and $1 \leq k \leq 55$. This table is inverted in the sense that $k$ increases down columns and $n$ varies along the columns. 


\section{Theorem 1.}

$$
R(n)=\sum_{k=0}^{\left(\begin{array}{c}
n \\
2
\end{array}\right)} k R(n, k)=\left(\begin{array}{l}
n \\
2
\end{array}\right) B_{n-1}\left(B_{n}-B_{n-1}\right)
$$

Proof. Choose a pair $\{x, y\}$. The number of partitions in which this pair appears in the same block is $B_{n-1}$. The number of partitions in which this pair appears in different blocks is the difference $B_{n}-B_{n-1}$. Thus in total, each pair contributes $B_{n-1}\left(B_{n}-B_{n-1}\right)$ to the sum. Since there are $\left(\begin{array}{c}n \\ 2\end{array}\right)$ ways to choose a pair, the proof is finished.

The average value of the Rand distance is thus

$$
\frac{R(n)}{\left(\begin{array}{c}
B_{n} \\
2
\end{array}\right)}=\frac{n(n-1) B_{n-1}\left(B_{n}-B_{n-1}\right)}{B_{n}\left(B_{n}-1\right)} .
$$

Since the Bell numbers grow exponentially,

$$
\frac{R(n)}{\left(\begin{array}{c}
B_{n} \\
2
\end{array}\right)} \sim n^{2} \frac{B_{n-1}}{B_{n}}
$$

which experimentally appears to be $\Theta(n \log n)$.

\subsection{Determining $R(n, k)$ for small values of $k$}

We now consider $R(n, k)$ for small values of $k$.

Clearly $R(n, 0)=0$.

Theorem 2. For all $n \geq 1$,

$$
R(n, 1)=\left(\begin{array}{l}
n \\
2
\end{array}\right) B_{n-2}
$$

Proof. The only way that the Rand distance can be 1 is if there is a block $\{x, y\}$ in one partition and two blocks $\{x\},\{y\}$ in the other, and all other blocks in one partition are present in the other. There are $\left(\begin{array}{l}n \\ 2\end{array}\right)$ ways to choose the pair and $B_{n-2}$ ways to determine the other blocks.

Corollary 1. The egf of the $R(n, 1)$ numbers is

$$
\sum_{n \geq 1} R(n, 1) \frac{z^{n}}{n !}=\frac{z^{2}}{2} B(z)=\frac{z^{2}}{2} e^{e^{z}-1} .
$$

Proof. Apply (2) with $k=2$.

The previous two results were warm-ups for the more technical results that follow. 
Theorem 3. For fixed $k$, there are non-negative integer constants $c_{k, j}$ such that, for all $n \geq 1$,

$$
R(n, k)=\sum_{j=\lceil(1+\sqrt{1+8 k}) / 2\rceil}^{2 k} c_{k, j}\left(\begin{array}{l}
n \\
j
\end{array}\right) B_{n-j} .
$$

Proof. Any two partitions $P$ and $Q$ will have a largest subpartition $X$ that is common to both $P$ and $Q$. Thus $\mathcal{R}(P, Q)=\mathcal{R}(P \backslash X, Q \backslash X)$.

In the sum above $j$ represents $n-|X|$, given that $\mathcal{R}(P, Q)=k$. The lower bound in the summation follows from the fact that the maximum Rand distance between two partitions of $n$ is $\left(\begin{array}{l}n \\ 2\end{array}\right)$ and thus $k \leq\left(\begin{array}{l}j \\ 2\end{array}\right)$. Solving the implied quadratic yields $j \geq(1+\sqrt{1+8 k}) / 2$, which gives us the lower bound. We hereafter use $\alpha=\lceil(1+\sqrt{1+8 k}) / 2\rceil$ for ease of reading.

For the upper bound, consider two partitions $P$ and $Q$ of an $j$-set that have no block in common, and have Rand distance $k$. We claim that $\geq\lceil j / 2\rceil$. Consider some arbitrary integer $x \in\{1,2, \ldots, j\}$. Since $P$ and $Q$ have no common blocks, there is some integer $y$ that is in the same block as $x$ in one partition, and in another block in the other partition. Thus we have $j$ distinct ordered pairs $(x, y)$, one for each different value of $x$. At least $\lceil j / 2\rceil$ of them have to be distinct as unordered pairs, and each such unordered pair contributes 1 to the Rand distance. Thus $k \geq\lceil j / 2\rceil$ as claimed. From this it follows that $j \leq 2 k$, which is the upper bound in the sum above.

Theorem 4. For all $n \geq 1$,

$$
R(n, 2)=6\left(\begin{array}{l}
n \\
3
\end{array}\right) B_{n-3}+6\left(\begin{array}{l}
n \\
4
\end{array}\right) B_{n-4} .
$$

Proof. Theorem 3 tells us that

$$
R(n, 2)=c_{2,3}\left(\begin{array}{l}
n \\
3
\end{array}\right) B_{n-3}+c_{2,4}\left(\begin{array}{l}
n \\
4
\end{array}\right) B_{n-4} .
$$

From the $k=2$ row of Table 2 we then have the following two equations.

$$
\begin{aligned}
& R(3,2)=6=c_{2,3}\left(\begin{array}{l}
3 \\
3
\end{array}\right) B_{0}+c_{2,4}\left(\begin{array}{l}
3 \\
4
\end{array}\right) B_{-1}=c_{2,3} \quad \text { and } \\
& R(4,2)=30=c_{2,3}\left(\begin{array}{l}
4 \\
3
\end{array}\right) B_{1}+c_{2,4}\left(\begin{array}{l}
4 \\
4
\end{array}\right) B_{0}=c_{2,3} 4+c_{2,4} .
\end{aligned}
$$

This system of equations can be solved to obtain $c_{2,3}=c_{2,4}=6$.

Corollary 2. The egf of the $R(n, 2)$ numbers is

$$
\sum_{n \geq 1} R(n, 2) \frac{z^{n}}{n !}=\left(z^{3}+\frac{z^{4}}{4}\right) B(z)=\left(z^{3}+\frac{z^{4}}{4}\right) e^{e^{z}-1} .
$$


In a similar fashion we can solve systems of linear equations to obtain the following theorems and corollaries.

Theorem 5. For all $n \geq 1$,

$$
R(n, 3)=\left(\begin{array}{l}
n \\
3
\end{array}\right) B_{n-3}+28\left(\begin{array}{l}
n \\
4
\end{array}\right) B_{n-4}+120\left(\begin{array}{l}
n \\
5
\end{array}\right) B_{n-5}+60\left(\begin{array}{l}
n \\
6
\end{array}\right) B_{n-6} .
$$

Corollary 3. The egf of the $R(n, 3)$ numbers is

$\sum_{n \geq 1} R(n, 3) \frac{z^{n}}{n !}=\left(\frac{z^{3}}{6}+\frac{7 z^{4}}{6}+z^{5}+\frac{z^{6}}{12}\right) B(z)=\left(\frac{z^{3}}{6}+\frac{7 z^{4}}{6}+z^{5}+\frac{z^{6}}{12}\right) e^{e^{z}-1}$.

Theorem 6. For all $n \geq 1$, the value of $R(n, 4)$ is

$24\left(\begin{array}{l}n \\ 4\end{array}\right) B_{n-4}+180\left(\begin{array}{l}n \\ 5\end{array}\right) B_{n-5}+1560\left(\begin{array}{l}n \\ 6\end{array}\right) B_{n-6}+2520\left(\begin{array}{l}n \\ 7\end{array}\right) B_{n-7}+840\left(\begin{array}{l}n \\ 8\end{array}\right) B_{n-8}$.

Corollary 4. The egf of the $R(n, 4)$ numbers is

$$
\sum_{n \geq 1} R(n, 4) \frac{z^{n}}{n !}=\left(z^{4}+\frac{3 z^{5}}{2}+\frac{13 z^{6}}{6}+\frac{z^{7}}{2}+\frac{z^{8}}{48}\right) B(z) .
$$

Intuitively, $c_{k, j}$ is the number of pairs of $j$-element set partitions with no common blocks that have Rand Distance $k$. We summarize the known values of $c_{k, j}$ in Table 3. Although we don't know the value of $c_{k, j}$ in general, we can determine a few specific infinite sequences, which are given in the next lemma.

Lemma 1. For all $k \geq 1$,

$$
c_{k, 2 k}=\frac{(2 k-1) !}{(k-1) !} \quad \text { and } \quad c_{k, \alpha}=R(\alpha, k) .
$$

Proof. For a pair of $2 k$ element set partitions $P$ and $Q$ to have Rand distance $k$ with no common blocks, the $2 k$ elements must be paired, and each pair of elements is a block in either $P$ or $Q$. Further, if $\{a, b\}$ is a block in set $P$ then set $B$ contains the singleton blocks $\{a\}$ and $\{b\}$ and vice versa. Since the order of the blocks doesn't matter, we can assume the blocks (pairs) are sorted by their smallest elements. So, for $i=1,2, \ldots, k$, once we have chosen the elements for blocks $1,2, \ldots, i-1$, the first element in block $i$ must be the smallest remaining element and there are $2 k-(2(i-1)+1)=2 k-2 i+1$ choices for the second element in block $i$. Thus the number of ways to pair the elements is

$$
\prod_{i=1}^{k}(2 k-2 i+1)=\frac{(2 k-1) !}{2^{k-1}(k-1) !}
$$

If we assume, without loss of generality, that a pair, say $\{a, b\}$, is in partition $P$, then there are $2^{k-1}$ unique ways to distribute the remaining pairs between $P$ and $Q$. So we have

$$
c_{j, 2 k}=\frac{(2 k-1) !}{2^{k-1}(k-1) !} 2^{k-1}=\frac{(2 k-1) !}{(k-1) !} .
$$




\begin{tabular}{|c|c|c|c|c|c|c|c|c|}
\hline$k \backslash j$ & 23 & 4 & 6 & 7 & 8 & 9 & 10 & 11 \\
\hline 1 & 1 & & & & & & & \\
\hline 2 & 6 & 6 & & & & & & \\
\hline 3 & 1 & 28120 & 60 & & & & & \\
\hline 4 & & 24180 & 1560 & 2520 & 840 & & & \\
\hline 5 & & 6210 & 1986 & 18900 & 63840 & 60480 & 15120 & \\
\hline 6 & & 1215 & 2780 & 28224 & 253246 & 1340640 & 2520000 & 1663200 \\
\hline 7 & & 60 & 2040 & 43365 & 463128 & 3998736 & 26878320 & 82328400 \\
\hline 8 & & 15 & 2610 & 38850 & 721728 & 8575200 & 74028240 & 554843520 \\
\hline 9 & & 10 & 1015 & 39060 & 778400 & 13061020 & 172444150 & 1568364600 \\
\hline 10 & & 1 & 465 & 28077 & 914480 & 17680572 & 270474480 & 3714220092 \\
\hline 11 & & & 150 & 23478 & 619416 & 19277748 & 407335320 & 6281694045 \\
\hline 12 & & & 35 & 13895 & 667450 & 19168422 & 482217540 & 10078945140 \\
\hline 13 & & & 45 & 4410 & 376040 & 17848152 & 529667460 & 12553128060 \\
\hline 14 & & & 15 & 1785 & 354060 & 13798458 & 530778780 & 15995950740 \\
\hline 15 & & & 1 & 1295 & 167664 & 11437644 & 477563400 & 16021896264 \\
\hline 16 & & & & 252 & 113764 & 7906059 & 431141400 & 17216673870 \\
\hline 17 & & & & 210 & 41832 & 5852700 & 315103995 & 15141561930 \\
\hline 18 & & & & 140 & 19614 & 3492426 & 275308740 & 14124874940 \\
\hline 19 & & & & 105 & 6020 & 2369304 & 174009780 & 11315379955 \\
\hline 20 & & & & 21 & 6930 & 1186227 & 146107962 & 9400242852 \\
\hline 21 & & & & 1 & 3500 & 609336 & 80801970 & 7071057840 \\
\hline 22 & & & & & 574 & 310662 & 60530130 & 5334533160 \\
\hline 23 & & & & & 840 & 190008 & 31267440 & 3888920970 \\
\hline 24 & & & & & 665 & 51303 & 25130325 & 2590267020 \\
\hline 25 & & & & & 476 & 41832 & 10882746 & 1799914809 \\
\hline 26 & & & & & 210 & 28476 & 6461280 & 1140678990 \\
\hline 27 & & & & & 28 & 25480 & 3015180 & 753854310 \\
\hline 28 & & & & & 1 & 7686 & 1926855 & 431506790 \\
\hline 29 & & & & & & 4104 & 1491300 & 290015550 \\
\hline 30 & & & & & & 2226 & 734820 & 169030620 \\
\hline 31 & & & & & & 3780 & 173610 & 110115390 \\
\hline 32 & & & & & & 2205 & 190575 & 50872635 \\
\hline 33 & & & & & & 1344 & 184905 & 33316140 \\
\hline 34 & & & & & & 378 & 134820 & 15268440 \\
\hline 35 & & & & & & 36 & 82152 & 12873861 \\
\hline 36 & & & & & & 1 & 21070 & 10432455 \\
\hline 37 & & & & & & & 16200 & 6565900 \\
\hline 38 & & & & & & & 15750 & 2310165 \\
\hline 39 & & & & & & & 14910 & 1281555 \\
\hline 40 & & & & & & & 13545 & 653169 \\
\hline 41 & & & & & & & 7245 & 1240470 \\
\hline 42 & & & & & & & 3270 & 824670 \\
\hline 43 & & & & & & & 630 & 574035 \\
\hline 44 & & & & & & & 45 & 214830 \\
\hline 45 & & & & & & & 1 & 104302 \\
\hline 46 & & & & & & & & 62205 \\
\hline 47 & & & & & & & & 103950 \\
\hline 48 & & & & & & & & 70455 \\
\hline 49 & & & & & & & & 74250 \\
\hline 50 & & & & & & & & 45045 \\
\hline 51 & & & & & & & & 21945 \\
\hline 52 & & & & & & & & 7095 \\
\hline 53 & & & & & & & & 990 \\
\hline 54 & & & & & & & & 55 \\
\hline 55 & & & & & & & & 1 \\
\hline
\end{tabular}

Table 3. Known values of $c_{k, j}$ for $2 \leq j \leq 11$. The bold value at the beginning of each row is $c_{k, \alpha}=R(\alpha, k)$. 
Since $B_{i}=0$ when $i<0, B_{0}=1$, and $\left(\begin{array}{l}i \\ i\end{array}\right)=1$,

$$
R(\alpha, k)=\sum_{j=\alpha}^{2 k} c_{k, j}\left(\begin{array}{c}
\alpha \\
j
\end{array}\right) B_{\alpha-j}=c_{k, \alpha} .
$$

Lemma 2. For all $j \geq 1$,

$$
c_{\left(\begin{array}{c}
j \\
2
\end{array}\right), j}=R\left(j,\left(\begin{array}{l}
j \\
2
\end{array}\right)\right)=1 .
$$

For all $j \geq 4$ :

$$
c_{\left(\begin{array}{l}
j \\
2
\end{array}\right)-1, j}=R\left(j,\left(\begin{array}{l}
j \\
2
\end{array}\right)-1\right)=\left(\begin{array}{l}
j \\
2
\end{array}\right) .
$$

For all $j \geq 5$ :

$$
c_{\left(\begin{array}{l}
j \\
2
\end{array}\right)-2, j}=R\left(j,\left(\begin{array}{l}
j \\
2
\end{array}\right)-2\right)=\left(\begin{array}{c}
\left(\begin{array}{c}
j-1 \\
2
\end{array}\right) \\
2
\end{array}\right) .
$$

For all $j \geq 2+x$ :

$$
c_{\left(\begin{array}{l}
j \\
2
\end{array}\right)-x, j}=R\left(j,\left(\begin{array}{l}
j \\
2
\end{array}\right)-x\right) .
$$

Proof. Omitted in this extended abstract.

\subsection{The numbers $R\left(n,\left(\begin{array}{l}n \\ 2\end{array}\right)-k\right)$ for small $k$}

We now consider the numbers at the bottom of the columns in Table 2. Clearly $R\left(n,\left(\begin{array}{l}n \\ 2\end{array}\right)\right)=1$ (the pair is $\{1,2 \ldots n\}$ and $\{1\}\{2\} \ldots\{n\}$ ).

Theorem 7. For all $n \geq 4$,

$$
R\left(n,\left(\begin{array}{l}
n \\
2
\end{array}\right)-1\right)=\left(\begin{array}{l}
n \\
2
\end{array}\right), \text { and } R(3,2)=6 .
$$

Proof. For $n \geq 4$, the two partitions are the full set $\{1,2, \ldots, n\}$ and the partition consisting of one pair and $n-2$ singleton sets.

Theorem 8. For all $n \geq 5$,

$$
R\left(n,\left(\begin{array}{l}
n \\
2
\end{array}\right)-2\right)=\left(\begin{array}{c}
n-1 \\
2 \\
2
\end{array}\right)=\frac{1}{8} n(n-1)(n-2)(n-3),
$$

and $R(3,3)=3, R(4,5)=24$. 
Proof. For $n \geq 5$ the two partitions are the full set $\{1,2, \ldots, n\}$ and the partition consisting of two pairs and $n-4$ singleton sets. The order of the two pairs does not matter so we have

$$
R\left(n,\left(\begin{array}{l}
n \\
2
\end{array}\right)-2\right)=\frac{1}{2}\left(\begin{array}{c}
n \\
2
\end{array}\right)\left(\begin{array}{c}
n-2 \\
2
\end{array}\right)
$$

which can be shown to be equal to the two values given in the statement of the theorem.

The numbers in Theorems 7 and 8 are a shifted versions of OEIS A000217 and OEIS A050534, respectively.

Theorem 9. For all $n \geq 6$,

$$
R\left(n,\left(\begin{array}{l}
n \\
2
\end{array}\right)-3\right)=\frac{1}{6}\left(\begin{array}{c}
n \\
2
\end{array}\right)\left(\begin{array}{c}
n-2 \\
2
\end{array}\right)\left(\begin{array}{c}
n-4 \\
2
\end{array}\right)+\left(\begin{array}{l}
n \\
3
\end{array}\right),
$$

and $R(4,3)=32, R(5,7)=60$.

Proof. For $n \geq 5$ the two partitions are either the full set $\{1,2, \ldots, n\}$ and the partition consisting of three pairs and $n-6$ singleton sets, or the full set $\{1,2, \ldots, n\}$ and the partition consisting of one triple and $n-3$ singleton sets.

Theorem 10. For fixed $k$ there is a constant $K_{k}$ such that $R\left(n,\left(\begin{array}{c}n \\ 2\end{array}\right)-k\right)$ is a polynomial of degree $2 k$ in $n$ for all $n \geq K_{k}$.

Proof. Omitted in this extended abstract.

\section{Acknowledgements}

We wish to thank Rod Canfield for helpful discussions.

\section{References}

1. E.R. Canfield, Engel's inequality for the Bell numbers, Journal of Combinatorial Theory, Series A, 72 (1995) 184-187.

2. V. Filkov and S. Skiena, Integrating Microarray Data by Consensus Clustering, International Journal on Artificial Intelligence Tools, 13(4): 863-880, 2004.

3. R.L. Graham, D.E. Knuth, and O. Patashnik, Concrete Mathematics, AddisonWesley, 1989.

4. L. Hubert, Comparing Partitions, Journal of Classification, 2 (1985) 193-218.

5. D.E. Knuth, The Art of Computer Programming, Volume 4: Combinatorial Algorithms, Part 1, Addison-Wesley, Reading, MA, 2011.

6. L. Moser and A. Wyman, An asymptotic formula for the Bell numbers, Transactions of the Royal Society of Canada III, 49 (1955) 49-54. 
7. A. O. Munagi, Set Partitions with Successions and Separations, Int. J. Math and Math. Sc. 2005, no. 3 (2005), 451-463.

8. W. Rand, Objective criteria for the evaluation of clustering methods, J. American Statistical Assoc., 66 (336): 846-850, 1971.

9. F. Ruskey, Simple combinatorial Gray codes constructed by reversing sublists, 4th ISAAC (International Symposium on Algorithms and Computation), Lecture Notes in Computer Science (LNCS), \#762 (1993) 201-208.

10. Richard R. Stanley, Enumerative Combinatorics, Volume 1 Wadsworth, 1986. 